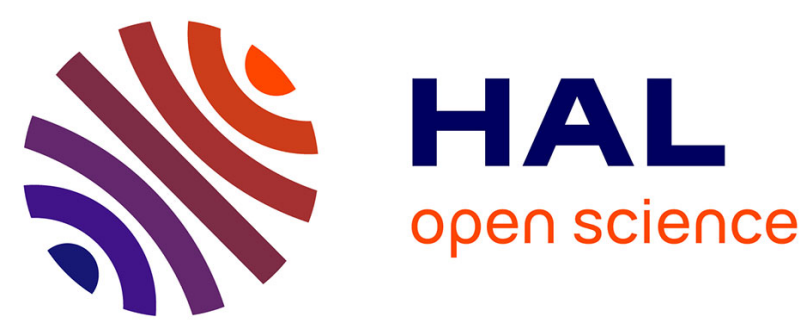

\title{
Broadband nonreciprocal acoustic scattering using a loudspeaker with asymmetric feedback
}

\author{
G. Penelet, V. Pagneux, G. Poignand, C. Olivier, Y. Aurégan
}

\section{To cite this version:}

G. Penelet, V. Pagneux, G. Poignand, C. Olivier, Y. Aurégan. Broadband nonreciprocal acoustic scattering using a loudspeaker with asymmetric feedback. Physical Review Applied, 2021, 16 (6), pp.064012. 10.1103/PhysRevApplied.16.064012 . hal-03468222

HAL Id: hal-03468222

https://hal-univ-lemans.archives-ouvertes.fr/hal-03468222

Submitted on 7 Dec 2021

HAL is a multi-disciplinary open access archive for the deposit and dissemination of scientific research documents, whether they are published or not. The documents may come from teaching and research institutions in France or abroad, or from public or private research centers.
L'archive ouverte pluridisciplinaire HAL, est destinée au dépôt et à la diffusion de documents scientifiques de niveau recherche, publiés ou non, émanant des établissements d'enseignement et de recherche français ou étrangers, des laboratoires publics ou privés. 


\title{
Broadband nonreciprocal acoustic scattering using a loudspeaker with asymmetric feedback
}

\author{
G. Penelet, ${ }^{*}$ V. Pagneux, G. Poignand, C. Olivier, and Y. Aurégan \\ Laboratoire d'Acoustique de l'Université du Mans (LAUM), \\ UMR-CNRS 6613, Institut d'Acoustique-Graduate School (IA-GS), CNRS, \\ Le Mans Université, Avenue O. Messiaen, 72085 Le Mans France
}

(Dated: November 22, 2021)

\begin{abstract}
This paper describes an acoustic system enabling to achieve asymmetric transmission over a broad frequency range. The basic elements of the system are a moving coil loudspeaker placed along the axis of a duct, and a microphone flushed-mounted next to the front side of the loudspeaker. The loudspeaker acts both as a mass-spring oscillator through which incident waves are reflected/transmitted, and as a sound re-emitter controlled by the microphone through a feedback loop. It is shown that the reciprocity of the resulting two-port is broken, and that it can be used to design a broadband asymmetric wave transmitter. The experimental results show that there exists a frequency band ranging from about $300 \mathrm{~Hz}$ up to $1500 \mathrm{~Hz}$ where an almost perfect transmission in the forward direction is observed whereas a weak (i.e., of about 10\%) transmission is achieved in the reverse direction. Owing to the broadbandness of this efficient and compact nonreciprocal scatterer, it is also shown through its response to incident pulses that it can be used as a sound trap.
\end{abstract}

\section{INTRODUCTION}

In the context of an intense research on acoustic metamaterials, a lot of work has been done on the development of nonreciprocal systems. One of the first systems proposed $[1,2]$ was based on the use of a sonic crystal combined with a nonlinear medium. Many other systems were subsequently designed in the last decade [3-13], and most of them rely on using nonlinear effects $[3,4,6-$ $8,10,11,13]$ to break the (usually inherent) reciprocity of acoustic systems. More generally, there is currently a large effort in developing nonreciprocal acoustic systems, with numerous potential applications (see ref. 14 for a recent review on this topic), but it is still challenging to achieve the design of simple, compact (sub-wavelength), and broadband systems demonstrating an efficient asymmetric transmission.

Active control/tuning of acoustic transducers or acoustic media is another possible way to achieve nonreciprocal propagation [7, 15-20]. In particular, the idea of using systems made with moving coil loudspeakers controlled by an active feedback or by an electric shunt [21] has already been used for the design of acoustic metamaterials such as PT-symmetric systems [22], reconfigurable acoustic resonators [23], or systems achieving perfect transmission through disorder [24].

In this paper, we describe a system which relies on a moving coil loudspeaker and an analog feedback loop circuit, so as to act as an asymmetric wave transmitter. In the following, the term asymmetric wave transmitter (AWT) is employed to define an acoustic scatterer which achieves perfect transmission (and zero reflection) of incident waves in the forward direction, but which also achieves total reflection without transmission in the reverse direction. Systems sharing similar target-properties

* guillaume.penelet@univ-lemans.fr are also termed as acoustic isolators $[5,6]$, or acoustic diodes $[1,4,9,11]$ in other studies. The present system has the advantages of being simple, compact, linear and to achieve nonreciprocal behavior within a broad frequency range. The basic principles of the device are described in Sec.II, where it is presented how an asymmetrically located microphone connected to a feedback loop can be used to break the reciprocity and to make the system a broadband AWT. A prototype AWT is presented in Sec.III, as well as the experimental test bench, which consists of the AWT connected to ducts at both sides so as to characterize the transmission/reflection of plane waves impinging either on one side or on the other side. The experimental results are presented in Sec.IV, where the measured scattering coefficients are shown in good agreement with theory. The results notably show that the nonreciprocity factor (defined as the ratio of transmission coefficients) is larger than $25 \mathrm{~dB}$ within a frequency band ranging from $310 \mathrm{~Hz}$ up to $1460 \mathrm{~Hz}$. The efficiency and the broadbandness of the AWT is enlightened by means of its response to short pulses of sound impinging on either side of the system, confirming that the incoming wave is either blocked or transmitted through the AWT.

\section{BASIC PRINCIPLES OF THE SYSTEM}

A schematic drawing of the system studied in this paper is presented in Fig.1. As shown in Fig.1(a), it consists of a rigid disk that can oscillate along the axis of a duct, thanks to some elastic suspensions attached to the inner walls. The duct is filled with a compressible fluid (density $\left.\rho_{0}\right)$, and acoustic waves can propagate on either side of the mass-spring system. As we consider frequencies below the first cut-off frequency of the duct, only the planar mode is propagating along the axis (non-planar modes are evanescent).

When the feedback loop is switched off, the reflec- 
(a)
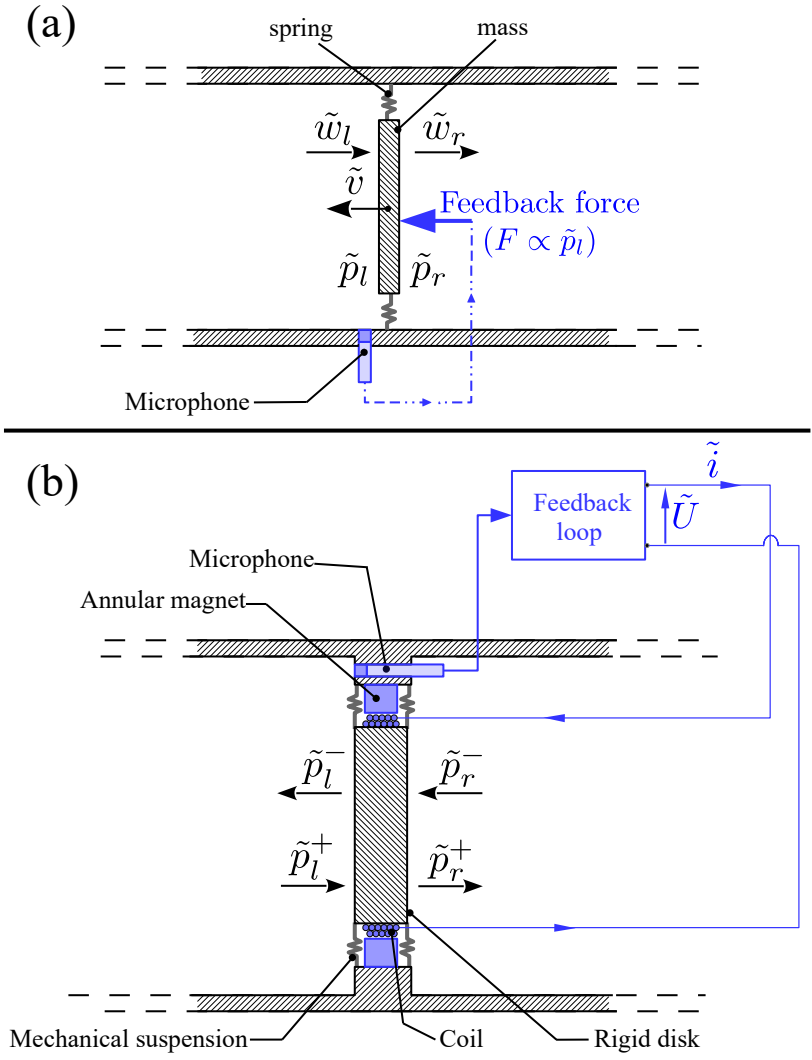

FIG. 1. Schematic drawing of the AWT. The notation $\tilde{p}_{l}$, or $\tilde{p}_{r}$, is used to describe the complex amplitude of the acoustic pressure at the left or the right side of the AWT, while $\tilde{p}_{l, r}^{+}$or $\tilde{p}_{l, r}^{-}$refer to the complex amplitude of a pressure wave propagating either rightwards $(+)$ or leftwards $(-)$; therefore one has $\tilde{p}_{l}=\tilde{p}_{l}^{+}+\tilde{p}_{l}^{-}$. The notation $\tilde{w}_{l, r}$ refers to the complex amplitudes of the volume velocity at any side of the AWT, and $\tilde{v}$ is the velocity of the oscillating disk.

tion/transmission properties of the mass-spring system can be described by a scattering matrix, which is symmetric and reciprocal. When the feedback loop is switched on, the specificity of the system relies on the fact that the disk is driven by a force which is proportional to the acoustic pressure $p_{l}$ at the left side, see Fig. 1(a). As will be shown in the following, this feedback force breaks the symmetry and the reciprocity of the two-port, which is a necessary condition to make it an AWT.

One mean of making the device of Fig.1(a) is to use an electrodynamic transducer connected through a feedback-loop to a microphone (giving access to $p_{l}$ ), as depicted in Fig.1(b). The electrodynamic transducer considered in this study is based upon the same principles than the ones of standard moving coil loudspeakers, although its geometry is slightly different ; standard loudspeakers have an electromagnet system placed behind a moving membrane, while the loudspeaker used here has identical front and rear sides. The mechanical part is made of a light and rigid disk attached to an annular magnet through lateral suspensions. A coil is wounded around the disk, and the annular magnet imposes a uniform magnetic field $B$ oriented radially through the coil. Therefore, if the coil of length $\ell$ is supplied by a time varying current, $i(t)$, then an axial electrodynamic force $F(t)=B \ell i(t)$ causes the coil (and thus the disk) to move back and forth. Moreover, any axial motion of the disk with a velocity $v(t)$ gives rise to a back-electromotive force that induces a voltage difference $B \ell v(t)$ at the coil terminals.

As a result, the equations describing the motion of the system (see Fig.1) write as

$$
\begin{aligned}
\tilde{U} & =\left(R_{e}+j \omega L_{e}\right) \tilde{i}+B \ell \tilde{v}, \\
Z_{m} \tilde{v} & =\left(\tilde{p}_{r}-\tilde{p}_{l}\right) S_{m}+B \ell \tilde{i},
\end{aligned}
$$

where the time varying quantities are described in the frequency domain through their complex amplitudes $\tilde{\xi}(\omega)$ defined as $\xi(t)=\Re\left(\tilde{\xi} e^{j \omega t}\right)$ where $\xi$ either refers to a voltage $U$, a current $i$, a pressure $p$ or a velocity $v$, while $\omega$ stands for the angular frequency and $j^{2}=-1$. In Eqs. (1-2), $S_{m}$ denotes the surface area of the disk, $R_{e}$ and $L_{e}$ stand for the electrical resistance and the inductance of the coil, and the mechanical impedance $Z_{m}$ of the massspring system writes as

$$
Z_{m}=j \omega M_{m}+R_{m}+\frac{1}{j C_{m} \omega}
$$

where $M_{m}$ denotes the mass of the disk, while $C_{m}$ and $R_{m}$ stand for the compliance and the mechanical resistance of the suspensions. Moreover, as illustrated in Fig.1(b), a feedback circuit imposes that the voltage assigned to the loudspeaker depends on the pressure $\tilde{p}_{l}$, which can be written as

$$
\tilde{U}=\tilde{H} \times \tilde{p}_{l},
$$

where $\tilde{H}(\omega)$ stands for the transfer function of the feedback loop. The acoustic volume velocities $\tilde{w}_{l, r}$ at both sides of the system are defined accordingly with Fig.1(a), so that the continuity of the volume flow rate leads to the additional relation $\tilde{w}_{l}=\tilde{w}_{r}=-\tilde{v} S_{m}$. Introducing the electrical impedance of the coil as $Z_{e}=R_{e}+j \omega L_{e}$, the equations above can be re-arranged to express the transfer matrix $\mathbf{T}$ of the two-port as:

$$
\mathbf{T}=\left(\begin{array}{cc}
1-\alpha & -\beta-j X \\
0 & 1
\end{array}\right)
$$

such that

$$
\left(\begin{array}{c}
\tilde{p}_{r} \\
\tilde{w}_{r}
\end{array}\right)=\mathbf{T} \times\left(\begin{array}{c}
\tilde{p}_{l} \\
\tilde{w}_{l}
\end{array}\right),
$$

with

$$
\begin{aligned}
\alpha & =\frac{\tilde{H} B \ell}{Z_{e} S_{m}}, \\
\beta & =\frac{R_{m}}{S_{m}^{2}}+\frac{(B \ell)^{2}}{Z_{e} S_{m}^{2}}, \\
j X & =\frac{1}{S_{m}^{2}}\left(j \omega M_{m}+\frac{1}{j \omega C_{m}}\right) .
\end{aligned}
$$


The coefficient $\alpha$ describes the impact of the feedback loop ( $\alpha=0$ if the feedback loop is switched off). The upper-right coefficient in $\mathbf{T}$, namely $-\beta-j X$, corresponds to the acoustic impedance of the AWT; $\beta$ mostly describes the losses due both to viscous friction in the mechanical suspensions and to the dissipation caused by Joule effect in the coil (note that as $Z_{e}=R_{e}+j L_{e} \omega$, $\beta$ also includes a reactive part due to the coil inductance $L_{e}$ ). The coefficient $X$ represents the acoustical reactance of the mass-spring system, which vanishes if $\omega=\omega_{s}=\left(M_{m} C_{m}\right)^{-1 / 2}$.

Introducing the characteristic impedance of the duct

$$
Z_{c}=\frac{\rho_{0} c_{0}}{S_{d}}
$$

where $S_{d}$ stands for the cross-sectional area of the duct and $c_{0}$ is the speed of sound in the fluid, the volume velocities at both sides of the system can be written as $\tilde{w}_{l, r}=\left(\tilde{p}_{l, r}^{+}-\tilde{p}_{l, r}^{-}\right) / Z_{c}$. So, from the T-matrix of Eq.(5), the relationship between incident and outgoing pressure waves can be obtained through the scattering matrix as

$$
\left(\begin{array}{c}
\tilde{p}_{r}^{+} \\
\tilde{p}_{l}^{-}
\end{array}\right)=\left(\begin{array}{cc}
\mathcal{T}^{+} & \mathcal{R}^{-} \\
\mathcal{R}^{+} & \mathcal{T}^{-}
\end{array}\right)\left(\begin{array}{l}
\tilde{p}_{l}^{+} \\
\tilde{p}_{r}^{-}
\end{array}\right)
$$

where the transmission and reflection coefficients are given by:

$$
\begin{aligned}
\mathcal{T}^{+} & =\frac{2(1-\alpha) Z_{c}}{(2-\alpha) Z_{c}+\beta+j X}, \\
\mathcal{T}^{-} & =\frac{2 Z_{c}}{(2-\alpha) Z_{c}+\beta+j X}, \\
\mathcal{R}^{+} & =\frac{\beta+j X+\alpha Z_{c}}{(2-\alpha) Z_{c}+\beta+j X}, \\
\mathcal{R}^{-} & =\frac{\beta+j X-\alpha Z_{c}}{(2-\alpha) Z_{c}+\beta+j X} .
\end{aligned}
$$

Therefore, it is shown here that as far as the feedback loop is switched on, i.e. $\alpha \neq 0$, the reciprocity of the system is broken since $\operatorname{det} \mathbf{T} \neq 1$ and $\mathcal{T}^{+} \neq \mathcal{T}^{-}$. This gives a chance to make an AWT if the transfer function $\tilde{H}$ of the feedback loop is chosen adequately.

In the following, attention is focused on the design of a system operating beyond the mechanical resonance, based on the following simple considerations. Should the angular frequency be such that $\omega \gg \omega_{s}$, then $j X \approx$ $j \omega M_{m} / S_{m}^{2}$ (i.e., above resonance the mass-spring system is governed by inertia). Moreover, if the voice coil inductance $L_{e}$ is neglected (only here for the sake of argument), then the parameter $\alpha$ writes as $(\tilde{H} B \ell) /\left(R_{e} S_{m}\right)$. Then having the feedback transfer function such that its output is a time derivative of the input with a controlled gain, namely if $\tilde{H} \propto j \omega$, allows us to use the feedback loop to control inertia effects and more precisely to make so that $\alpha Z_{c}=-j X$. Furthermore, if both assumptions that $\omega \gg S_{m}^{2} Z_{c} / M_{m}$ and $\omega \gg \beta S_{m}^{2} / M_{m}$ are valid then
TABLE I. Electromechanical parameters of the loudspeaker.

\begin{tabular}{lcc}
\hline \hline Parameter & Notation & Value \\
\hline Force factor & $B \ell$ & $5.6 \mathrm{~T} . \mathrm{m}$ \\
DC coil resistance & $R_{e}$ & $6.54 \Omega$ \\
Coil inductance & $L_{e}$ & $0.410^{-3} \mathrm{H}$ \\
Compliance of suspensions & $C_{m}$ & $0.5110^{-3} \mathrm{~m} . \mathrm{N}^{-1}$ \\
Resistance of suspensions & $R_{m}$ & $0.59 \mathrm{~N} . \mathrm{s.m}^{-1}$ \\
Moving mass & $M_{m}$ & $4.810^{-3} \mathrm{~kg}$ \\
Surface of the disk & $S_{m}$ & $3710^{-4} \mathrm{~m}^{2}$ \\
\hline \hline
\end{tabular}

it comes down from Eqs.(12) that the choice of a transfer function

$$
\tilde{H}=-j \omega \times \Gamma
$$

with

$$
\Gamma=\frac{M_{m} R_{e}}{B \ell S_{m} Z_{c}},
$$

leads to $\left|\mathcal{T}^{+}\right| \approx 1,\left|\mathcal{T}^{-}\right| \approx 0,\left|\mathcal{R}^{+}\right| \approx 0$, and $\left|\mathcal{R}^{-}\right| \approx 1$, which corresponds to the target scattering coefficients of a perfect acoustic AWT. It is therefore anticipated here that an AWT can be designed, which would be efficient within a potentially broad frequency range bounded down by the resonance frequency $\omega_{s}$ of the mass-spring oscillator, and bounded up by the plane wave approximation in the duct (i.e. $\omega<1.841 c_{0} / R_{d}$, where $R_{d}$ stands for the inner radius of the duct [25]). The experimental proof-of-concept of such a system is presented in the following.

\section{EXPERIMENTAL SET-UP.}

\section{A. Description of the AWT}

Two photographs of the AWT are presented in Fig.2. The loudspeaker is a cylindrical block with an outer radius of $45 \mathrm{~mm}$ and a thickness of $13 \mathrm{~mm}$, which is flushmounted within a cylindrical PVC support $(125 \mathrm{~mm}$ in outer diameter, $15 \mathrm{~mm}$ in thickness). The loudspeaker unit is a patented system [26] which, as explained in the previous section, consists of an annular permanent magnet equipped with the moving part of the system, namely the coil and the rigid disk attached to the magnetic ring through elastic suspensions. As shown in Fig.2, a measurement microphone (model GRAS 46BE) is flushmounted on the front side of the PVC support so as to provide the input for the feedback loop of the AWT unit.

The transducer is first characterized experimentally using standard methods of loudspeaker characterization based on the measurement of its electrical impedance as a function of the driving frequency [27]. The measured electromechanical parameters of the loudspeaker unit are given in Tab.I.

The AWT unit of Fig. 2 is built so as to be connected to standard PVC ducts with an outer diameter of $125 \mathrm{~mm}$ 

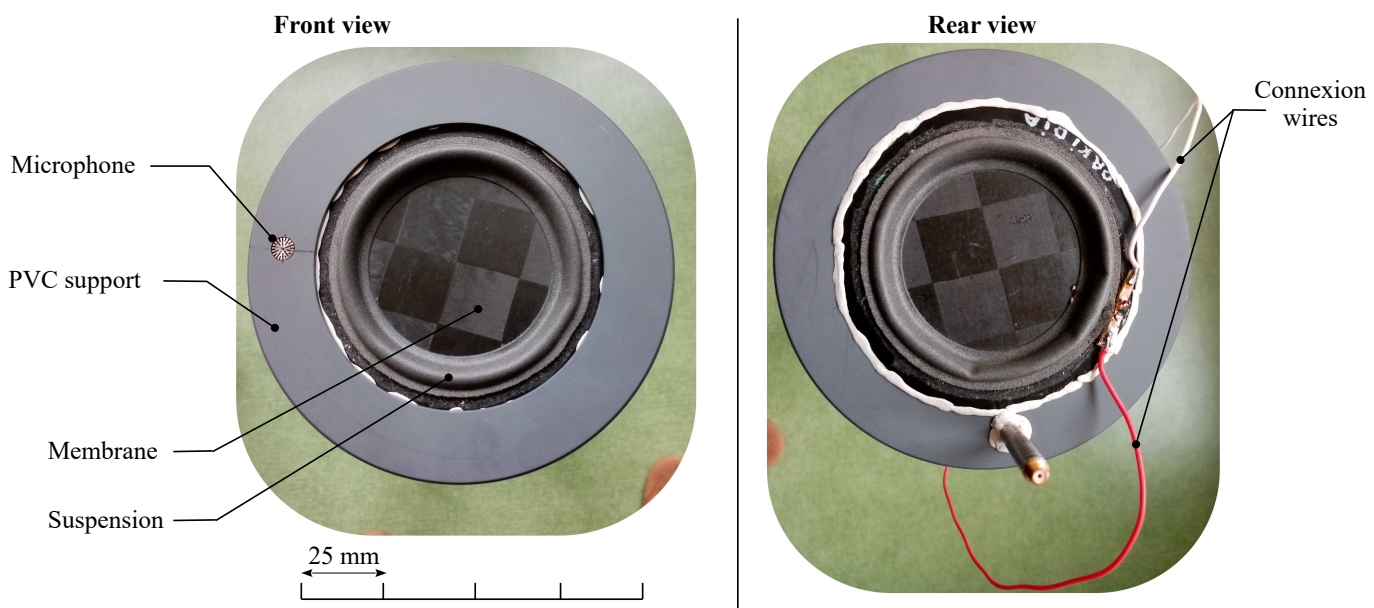

FIG. 2. Photographs of the asymmetric wave transmitter (AWT)

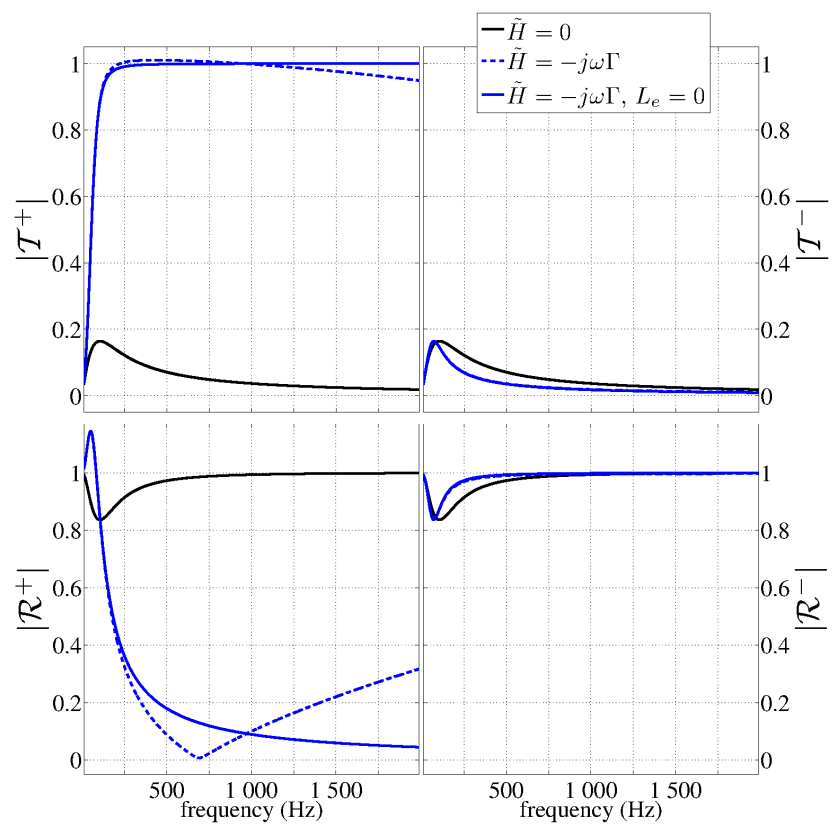

FIG. 3. Moduli of the scattering matrix coefficients of the AWT as functions of frequency, calculated using Eqs.(12) and using the loudspeaker parameters of Tab.I. Calculations are performed either without the feedback loop $(\tilde{H}=0$, black lines), or with a feedback transfer function defined accordingly with Eq.(13). For this latter case, the coefficients are plotted either with $L_{e}=0.4 \mathrm{mH}$ (dashed blue lines), or with a vanishing inductance $L_{e}=0 \mathrm{mH}$ (solid blue lines).

and an inner radius $R_{d}=58.5 \mathrm{~mm}$. Hence, the characteristic impedance $Z_{c}$ of the surrounding ducts is also known and the scattering matrix coefficients can be calculated as functions of the frequency using Eqs. (12). In Fig. 3, the calculated moduli of these coefficients are plotted as functions of the frequency, for both cases of $\tilde{H}=0$ (no feedback loop, black lines) and of $\tilde{H}=-j \omega \times \Gamma$ (blue lines). For this latter case, the coefficients are plotted either with the measured value of $L_{e}$, namely $L_{e}=0.4$ $\mathrm{mH}$ (dashed blue lines), or with a vanishing inductance $L_{e}=0 \mathrm{mH}$ (solid blue lines). Without feedback, $\alpha=0$ and the results confirm that the two-port is reciprocal with $\mathcal{T}^{+}=\mathcal{T}^{-}$. The maximum transmission is achieved around the resonance frequency of the mass-spring system, $f_{s} \approx 102 \mathrm{~Hz}$, and the corresponding transmission does not exceed a value of 0.2 ; such a low transmission around resonance is due to a significant damping (both mechanical and electrical) of the mass-spring system. Next, if the feedback loop is switched on, the results obtained while discarding the inductance of the voice coil (solid blue lines) confirm that the targeted properties of an AWT are observed beyond the resonance of the system, since the coefficients $\left|\mathcal{T}^{+}\right|$and $\left|\mathcal{R}^{-}\right|$tend towards unity, while both $\left|\mathcal{T}^{-}\right|$and $\left|\mathcal{R}^{+}\right|$gradually decrease as the frequency increases. It is worth noting, however, that if the coil inductance $L_{e}$ is taken into account then the scattering of waves by the system deviates from that of an ideal AWT: as shown by the curves with dashed blue lines (compared to those with solid lines) in Fig.3, both left-sided coefficients $\left|\mathcal{T}^{+}\right|$and $\left|\mathcal{R}^{+}\right|$depend significantly on the coil inductance, and some kind of low-pass filtering in the transmission of a wave impinging from the left is caused by the impact of $L_{e}$ on the dynamics of the loudspeaker. Nonetheless, the theoretical results of Fig.3 appear as convincing enough for setting-up an experimental proof of concept, which is described below.

\section{B. Experimental test-bench}

A schematic drawing of the experimental test-bench used for the measurements of the scattering matrix of the AWT is shown in Fig. 4. The AWT is tightly connected on each side to standard PVC ducts $(125 \mathrm{~mm}$ in outer diameter), which themselves are connected by 


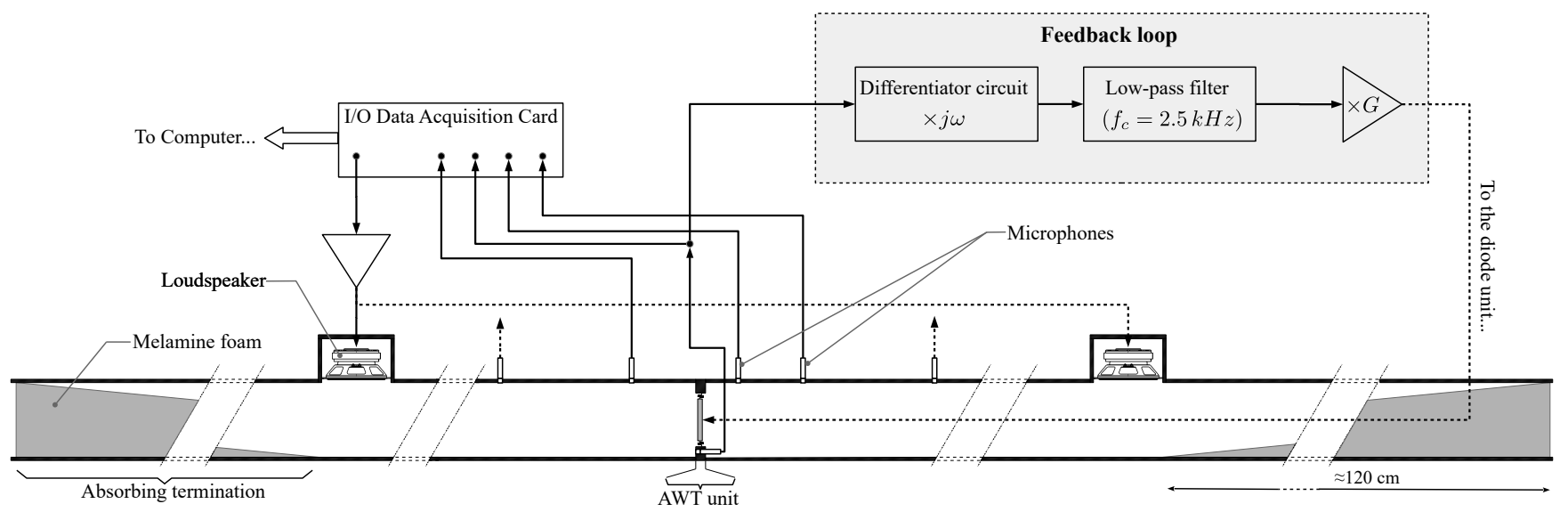

FIG. 4. Scale drawing of the experimental test-bench

means of a T-branch to both an absorbing termination and an acoustic source (model Fostex FF-105WK). Each absorbing termination is made up of a cylindrical PVC duct, about $120 \mathrm{~cm}$ in length, within which a cylindrical piece of melamine foam is inserted. The piece of foam is cut along its length so as to form a dihedron (as in standard acoustic wedge panels used for anechoic rooms) and to promote a weak reflection of acoustic waves by the termination. A standard two-source, multiple microphone method is used to measure the scattering matrix of the AWT. This method consists of making one set of measurements with each source, and using two pairs of microphones flush mounted at both sides of the two-port [28]. A simple post-processing of raw data enables to determine the amplitude and phase of counter-propagating waves at both sides of the system, and hence the scattering matrix of the two-port. The accuracy of such a method strongly depends on the relative calibration (both in amplitude and phase) of the microphones and on their spacing [29]. In the present device, we used $1 / 4$ in. measurement microphones (model GRAS 46BE connected to a 4 channels power module GRAS 12AX) which were first calibrated with each other using a small cavity coupler, and then flush-mounted along each duct. All microphones were connected to a I/O data acquisition card (model National Instrument USB 4431), which was also used for the generation of the signal provided to each source (a harmonic signal with a step by step increasing frequency was used for the measurements of transfer functions). As the accuracy of the measurements within a given frequency range is also related to the distance between the two microphones forming a pair, several distances were used in this study, namely 10,30 or 70 centimeters: this allowed to perform sufficiently reliable measurements of the scattering matrix coefficients for a frequency ranging from $70 \mathrm{~Hz}$ up to a maximum of $1.5 \mathrm{kHz}$, which is slightly below the cut-off frequency of the ducts (namely around $1750 \mathrm{~Hz}$ ).

The feedback loop circuit is also schematically pre- sented in Fig.4. As explained earlier, see Eq.(13), it is desired that the voltage supplied to the loudspeaker be proportional to $\dot{p}_{l}$, where $\dot{p}_{l}$ is the time derivative of the acoustic pressure measured by the control microphone of the AWT unit. The feedback loop circuit includes a differentiator circuit made with an operational amplifier [30] providing an output signal proportional to $\dot{p}_{l}$, and an audio power amplifier which is used to control the gain so as to reach the target value of $\Gamma$ given in Eq. (14).

It is worth mentioning that the presence of a positive feedback can lead to self-oscillations, a phenomenon commonly known as the audio feedback in public address systems and audio engineering applications. Such selfoscillations with a frequency of a few $\mathrm{kHz}$ were indeed observed in the present device when the gain of the audio amplifier, $G$, was set too high. Therefore, a passive low-pass $1^{\text {st }}$ order filter with a cut-off frequency of 2.5 $\mathrm{kHz}$ was also included in the feedback circuit to prevent from such undesired self-oscillations, or more precisely to push the limits in terms of a threshold gain giving rise to self-oscillations.

\section{EXPERIMENT RESULTS.}

\section{A. Scattering matrix coefficients}

The moduli and phases of the reflection/transmission coefficients of the AWT are presented in Fig.5 as functions of the frequency. Those measurements were performed for different values of the gain $G$ of the audio amplifier, and the corresponding feedback loop transfer function $\tilde{H}_{\text {exp }}=\tilde{U} / \tilde{p}_{l}$ was also measured. By measuring the slope of $\left|\tilde{H}_{\text {exp }}\right|$ which increases (almost) linearly with $\omega$, it was therefore possible to evaluate the experimental value of the total gain, with regards to the target value of $\Gamma$ defined in Eq.(14). The results obtained for the scattering matrix coefficients are plotted with solid lines in Fig.5 for different cases, namely without a feedback 

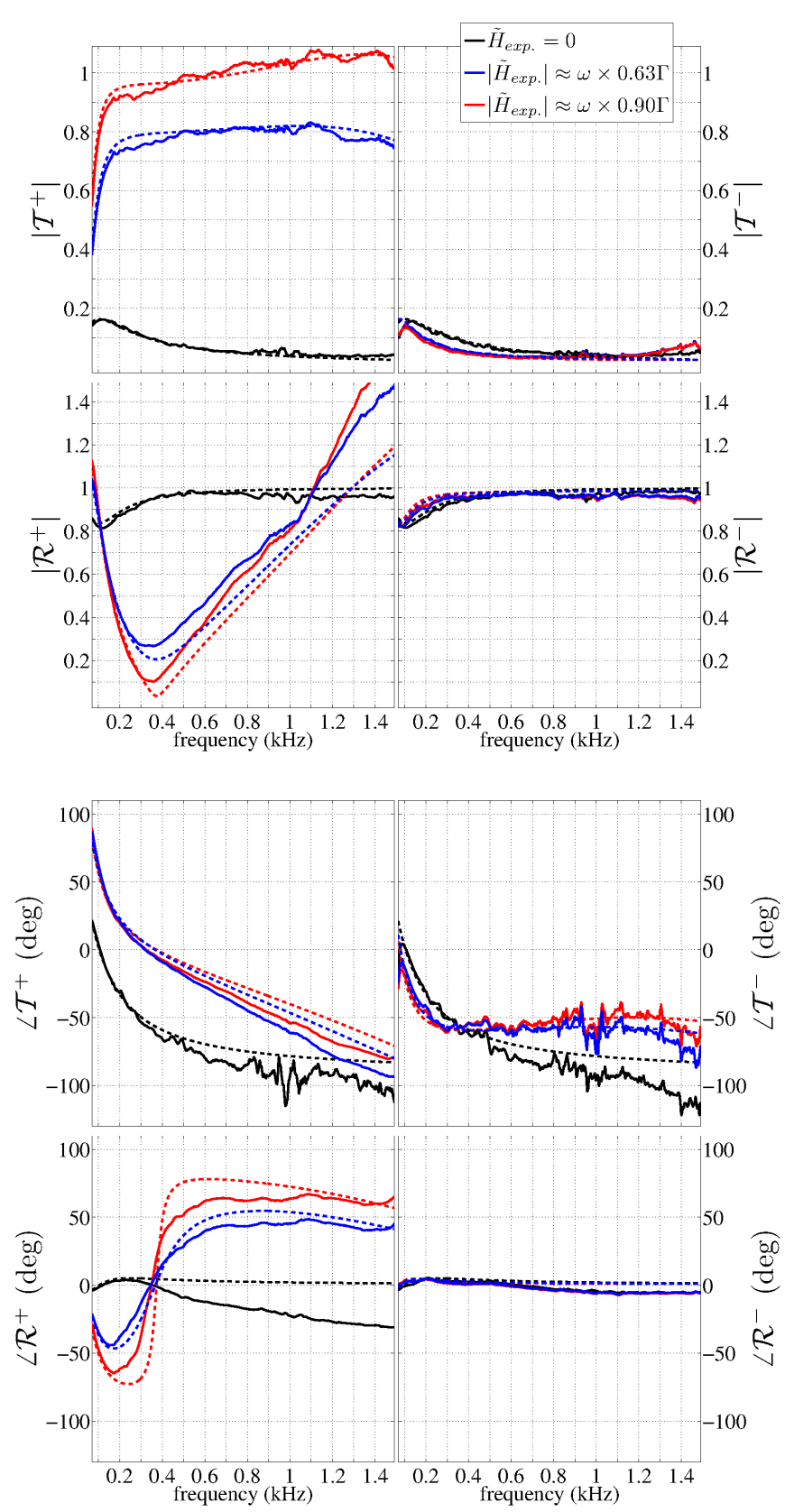

FIG. 5. Moduli (upper graphs) and phases (lower graphs) of the scattering matrix coefficients of the AWT as functions of the frequency and for different values of the feedback loop's transfer function $\left(\tilde{H}_{\text {exp }}\right)$. Solid lines correspond to the scattering matrix coefficients measured using the multiple microphones method, while dashed lines correspond to those calculated using Eqs.(12), in which the feedback loop parameter defined in Eq.(7) is evaluated from the measured transfer function of the feedback loop.

loop (black lines), and for two additional cases such that $\left|\tilde{H}_{\text {exp }}\right| \approx 0.63 \Gamma \times \omega$ (blue lines) or $\left|\tilde{H}_{\text {exp }}\right| \approx 0.90 \Gamma \times \omega$ (red lines). The target value of $\left|\tilde{H}_{e x p}\right|=\Gamma \times \omega$ could not be achieved in this system because self-oscillations appeared for values of the slope of $\left|\tilde{H}_{\text {exp }}\right|$ exceeding $0.96 \Gamma$. How- ever, the results show that even if the target value of $\Gamma$ is not perfectly achieved, the feedback enables to break the reciprocity of the two-port, and in particular it is possible to make $\left|\mathcal{T}^{+}\right|$approach unity while $\left|\mathcal{T}^{-}\right|$approaches zero over a large frequency range (see curves with solid red lines). Since the feedback includes a low pass-filter with a cut-off frequency of $2.5 \mathrm{kHz}$, but also since both the differentiator circuit and the power amplifier are not ideal components, the measured feedback transfer function actually deviates from the ideal one, $\tilde{H}=-j \omega \times \Gamma$. The measured transfer functions $\tilde{H}_{\text {exp }}(\omega)$ are presented in Fig. 7 in the appendix, and it is shown that their modulus do not perfectly increase linearly with $\omega$, while their phase depend on frequency (rather than being equal to $-\pi / 2$ ). Those measured transfer functions can be used to evaluate the experimental slopes relative to the ideal one, $\Gamma$. But more importantly, the measured $\tilde{H}_{\exp }(\omega)$ can be used as an input for the prediction of the scattering matrix coefficients using an experimental value of the parameter $\alpha=\tilde{H}_{e x p} B \ell /\left(Z_{e} S_{m}\right)$ in the expressions of the S-matrix coefficients defined in Eqs.(12). These calculated coefficients are plotted with dashed lines in Figs. 5 , and as a whole they show a good agreement with experimental data.

From a general standpoint, both experimental and theoretical results of Fig.5 show that the initial goal of designing an AWT over a large frequency range is possible since the targeted transmissions of $\left|\mathcal{T}^{+}\right| \approx 1$ and $\left|\mathcal{T}^{-}\right| \approx 0$ are roughly achieved. More precisely, the results show that the nonreciprocity factor (defined as the ratio of $\left|\mathcal{T}^{+}\right|$over $\left|\mathcal{T}^{-}\right|$) is larger than $25 \mathrm{~dB}$ within a frequency band ranging from $310 \mathrm{~Hz}$ up to $1460 \mathrm{~Hz}$. The experimental results also show that the left-sided coefficients are those which mostly depend on the feedback, as expected from the theoretical results of Fig. 3. As expected also, the case of a perfect broadband AWT cannot be achieved by the system, especially concerning the left-sided reflection coefficient $\left|\mathcal{R}^{+}\right|$which increases in the high frequency range and even exceeds unity (with subsequent risks of self-oscillating instabilities, depending on the acoustic load connected to the left-side of the system). This increase of $\left|\mathcal{R}^{+}\right|$is well-reproduced by the model, so that it can be partially explained by the finite value of $L_{e}$ and by the details of the feedback loop transfer function, but a discrepancy between experiments and theory is also observed as the frequency increases.

\section{B. Transmission/reflection of pulses through the AWT}

The results of Fig.5 clearly show that the system can be used as a broadband AWT, but the additional results presented hereafter aim at providing a more concrete and pictorial demonstration of its operation. To that purpose, the gain $G$ of the audio amplifier is fixed such that $\left|\tilde{H}_{e x p}\right| \approx 0.90 \Gamma \times \omega$ (giving rise to the scattering properties described by solid red lines in Fig.5), and the transmission/reflection of waves by the AWT is observed 

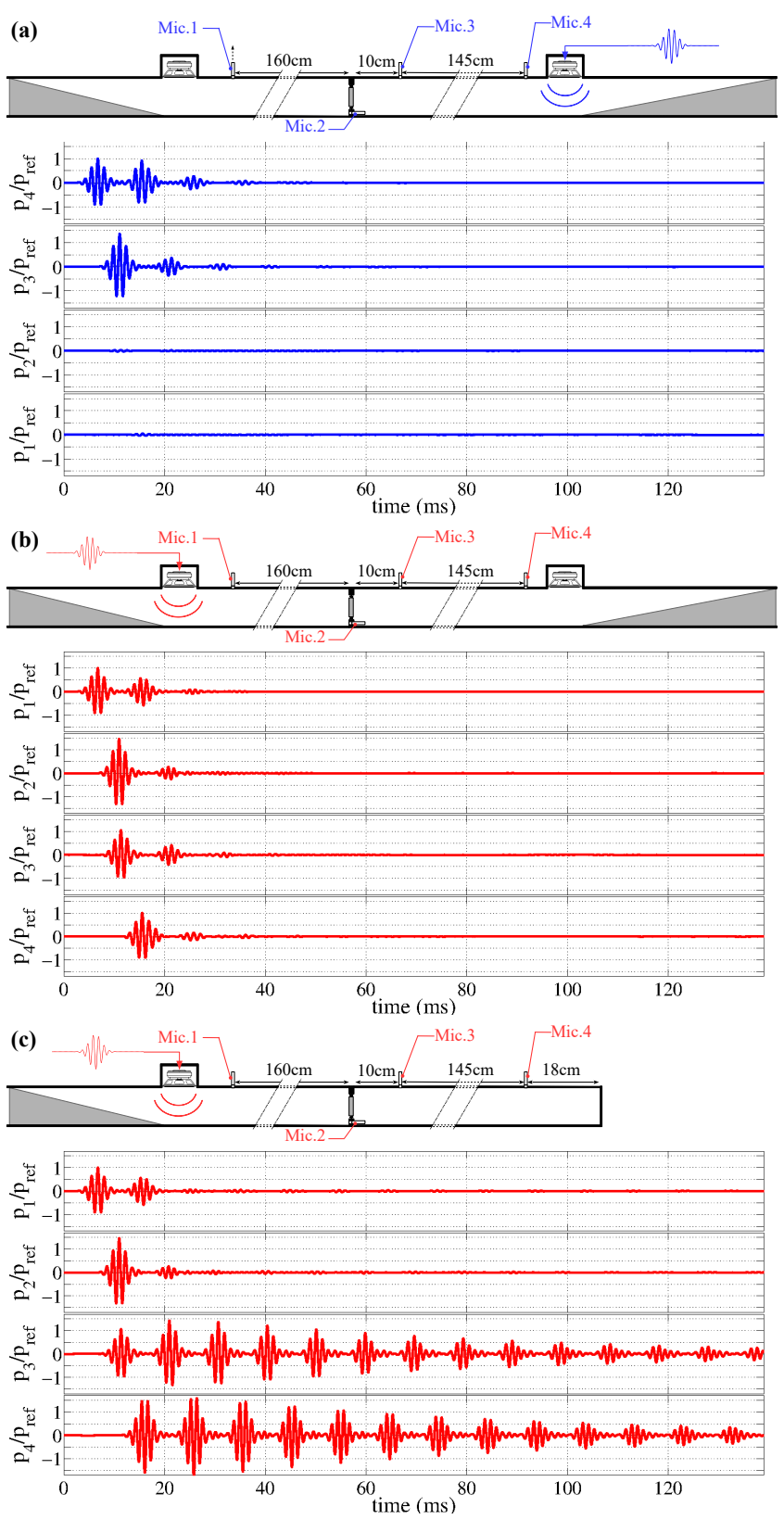

FIG. 6. Acoustic response of the AWT to a pulse. The response of the system is observed by means of four microphones flush mounted along the ducts. When the system is connected to weakly echoic terminations, its response is analyzed for both cases of an excitation from the right (a), or from the left (b). The system's response to a left-sided excitation when the the right side of the system is closed by a rigid plug is also presented in (c). The amplitudes of the microphone signals are all scaled by the same reference amplitude, $p_{\text {ref }}$, defined as the maximum peak amplitude of the incident pulse, namely $p_{\text {ref }}=\max \left(p_{4}\right)$ in $(\mathbf{a})$, and $p_{\text {ref }}=\max \left(p_{1}\right)$ in (b,c).

by using a broadband pulse wave which may impinge the system from the right or from the left side. The experimental results are presented in Fig.6. The input signal provided to (one of) the external loudspeaker(s) is a harmonic signal with a frequency fixed to $800 \mathrm{~Hz}$, whose amplitude is modulated by a Gaussian distribution function. The standard deviation $\sigma$ of this normal distribution function is fixed to $1 \mathrm{~ms}$, such that most of the energy of the pulse lies within a broad frequency range corresponding to the bandpass of the AWT. As depicted in Fig.6, two pairs of microphones are placed at both sides of the system (one of which includes the control microphone used as an input for the feedback loop), and their distance of separation is chosen large enough (namely about $1.5 \mathrm{~m}$ ) in order that the pulse signal of incident/reflected waves can be detected separately in the temporal data taken from each microphone.

For the two cases depicted in Figs.6(a) and (b), the AWT unit is connected to a poorly reflecting acoustic termination at both sides, and the incident wave is emitted either from the right side, see Fig.6(a), or from the left side, see Fig.6(b). The results of Fig.6(a) show that an incident pulse impinging from the right experiences total reflection (i.e., $\left|\mathcal{R}^{-}\right| \approx 1$ ) as clearly seen from the signal taken from microphone $\mathrm{n}^{\circ} 4$, and that almost nothing is transmitted through the AWT, as can be seen from time signals of microphones $\mathrm{n}^{\circ} 1$ and $\mathrm{n}^{\circ} 2$ : this is consistent with the expected result that $\left|\mathcal{T}^{-}\right| \approx 0$. The results of Fig.6(b) show that an incident pulse impinging from the left is transmitted to the right side of the AWT (i.e., $\left|\mathcal{T}^{+}\right| \approx 1$ ) and that that some reflection also occurs (as $\left.\left|\mathcal{R}^{+}\right| \neq 0\right)$.

Amusingly, the same experiment can be made when replacing the absorbing termination at right with a rigid plug, and the resulting acoustic response of the system to a left-sided impinging wave is shown in Fig.6(c). It is shown that the incident wave coming from the left side of the system is transmitted to the right, and the signals of mics. $\mathrm{n}^{\circ} 3$ and 4 clearly show subsequent reflections (and damping) of this pulse wave going back and forth on the right side of the AWT, without any transmission back to the left duct from which it was initially emitted. This configuration in Fig.6(c) corresponds to a sound trapping cavity able to capture the incident pulse.

\section{CONCLUSION.}

An asymmetric wave transmitter (AWT) has been proposed, based on a simple concept of asymmetric feedback which (to the authors' knowledge) had not been explored yet for the development of so-called acoustic diodes/isolators, and more generally of nonreciprocal systems. The experimental results show good agreement with the model and provide a robust proof of concept, since the system achieves the targeted asymmetric transmission over a frequency band of more than two octaves. The best merit of the proposed device probably owes to its simplicity, while the main drawback to its implementation is the management of potential instabilities due to the audio-feedback effect (a reason why low-pass filtering was employed here in the feedback loop). 
Improvements of the present system would be worth considering, notably regarding some modifications of the feedback transfer function so as to compensate for undesired effects related to the finite value of $L_{e}$, which has been identified here as a limiting factor. We also leave other potential improvements of the system, such as changing the feedback transfer function for the development of a system operating below or around resonance, to a future investigation. Extending the frequency range of operation of the AWT would also be interesting : this could be done by reducing the radius of the duct (with subsequent increase of its cut-off frequency) and by using a smaller loudspeaker. The loudspeaker used here is a custom made system having identical front and rear sides, but there is no obvious limitations in building such an AWT with standard moving coil loudspeakers. The extension of this work to large walls equipped with several AWT units and submitted to waves having an oblique incidence would also be worth considering. Also, it might be interesting to evaluate if such concepts used here for the propagation of sound in compressible fluids could be extended to asymmetric transmission of elastic waves.

\section{Appendix A: Feedback transfer function}

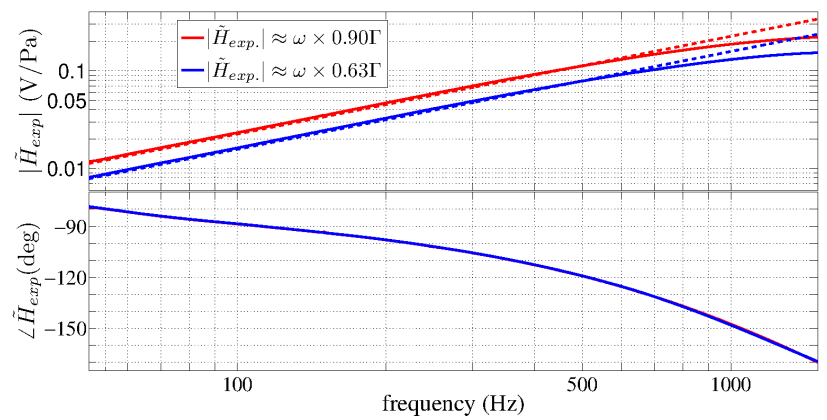

FIG. 7. Modulus and phase (solid lines) of the measured feedback transfer functions, $\tilde{H}_{e x p}$, associated to the experimental data presented in Fig.5. The straight dashed lines correspond to a linear fit of $\left|\tilde{H}_{\text {exp }}\right|$, which are used to evaluate the experimental slopes relative to the ideal one, $\Gamma$.

The feedback transfer function defined in Eq.(4) was measured on the experimental set-up. The moduli and phases of $\tilde{H}_{\text {exp }}$ are presented with solid lines in Fig. 7 as functions of the frequency, and for both cases presented in Fig.5 where the associated S-matrix coefficients are presented. These measured $\tilde{H}_{\text {exp }}(\omega)$ are used as an input for calculating the scattering matrix coefficients using an experimental value of the parameter $\alpha=\tilde{H}_{\text {exp }} B \ell /\left(Z_{e} S_{m}\right)$ in the expressions of the S-matrix coefficients defined in Eqs.(12) : this gives rise to the results with dashed lines in Fig.5. The straight curves with dashed lines in Fig.7 represent the two corresponding evaluations of the slopes (namely $0.63 \times \Gamma$ and $0.90 \times \Gamma$ ) of $\left|\tilde{H}_{\text {exp }}\right|$ relative to the predicted slope $\Gamma$ defined in (14) for the ideal case of a vanishing inductance.

\section{ACKNOWLEDGMENTS}

The authors gratefully acknowledge their colleague Antonin Novak as well as Bernard Nemoff (Orkidia Audio) for having kindly provided a prototype of the loudspeaker which was used here as the main element of the asymmetric wave transmitter. This research was funded by the French National Agency for Research (SelfiXs Project, grant agreement ANR-18-CE92-0001).
[1] B. Liang, B. Yuan, and J.-C. Cheng, Acoustic Diode: Rectification of Acoustic Energy Flux in OneDimensional Systems, Phys. Rev. Lett. 103, 104301 (2009).

[2] B. Liang, X. Guo, J. Tu, D. Zhang, and J. C. Cheng, An acoustic rectifier, Nature Mater. 9, 989-992 (2010).

[3] N. Boechler, G. Theocharis, and C. Daraio, Bifurcationbased acoustic switching and rectification, Nature Mater.
10, 665 (2011).

[4] X. Guo, Z. Lin, J. Tu, B. Liang, J. Cheng, and D. Zhang, Modeling and optimization of an acoustic diode based on micro-bubble nonlinearity, J. Acoust. Soc. Am. 133, 1119 (2013).

[5] R. Fleury, D. L. Sounas, C. F. Sieck, M. R. Haberman, and A. Alù, Sound Isolation and Giant Linear Nonreciprocity in a Compact Acoustic Circulator, Science 343, 
516 (2014).

[6] N. A. Estep, D. L. Sounas, J. Soric, and A. Alù, Magnetic-free non-reciprocity and isolation based on parametrically modulated coupled-resonator loops, Nature Phys. 10, 923-927 (2014).

[7] B. Popa and S. Cummer, Non-reciprocal and highly nonlinear active acoustic metamaterials, Nature Comm. 5, 3398 (2014).

[8] T. Devaux, V. Tournat, O. Richoux, and V. Pagneux, Asymmetric Acoustic Propagation of Wave Packets Via the Self-Demodulation Effect, Phys. Rev. Lett. 115, 234301 (2015).

[9] T. Biwa, H. Nakamura, and H. Hyodo, Experimental Demonstration of a Thermoacoustic Diode, Phys. Rev. Appl. 5, 064012 (2016).

[10] J.-G. Cui, T. Yang, and L.-Q. Chen, Frequencypreserved non-reciprocal acoustic propagation in a granular chain, Appl. Phys. Lett. 112, 181904 (2018).

[11] C. Fu, B. Wang, T. Zhao, and C. Q. Chen, High efficiency and broadband acoustic diodes, Applied Physics Letters 112, 051902 (2018).

[12] C. P. Wiederhold, D. L. Sounas, and A. Alù, Nonreciprocal acoustic propagation and leaky-wave radiation in a waveguide with flow, J. Acoust. Soc. Am. 146, 802 (2019).

[13] T. Devaux, A. Cebrecos, O. Richoux, V. Pagneux, and V. Tournat, Acoustic radiation pressure for nonreciprocal transmission and switch effects, Nature Comm. 10, $3292(2019)$.

[14] C. Rasmussen, L. Quan, and A. Alù, Acoustic nonreciprocity, J. Appl. Phys. 129, 210903 (2021).

[15] C. Shen, X. Zhu, J. Li, and S. A. Cummer, Nonreciprocal acoustic transmission in space-time modulated coupled resonators, Phys. Rev. B 100, 054302 (2019).

[16] Y. Chen, X. Li, H. Nassar, A. N. Norris, C. Daraio, and G. Huang, Nonreciprocal Wave Propagation in a Continuum-Based Metamaterial with Space-Time Modulated Resonators, Phys. Rev. Appl. 11, 064052 (2019).

[17] Y. Zhai, H.-S. Kwon, and B.-I. Popa, Active Willis metamaterials for ultracompact nonreciprocal linear acoustic devices, Phys. Rev. B 99, 220301 (2019).

[18] S. Karkar, E. De Bono, M. Collet, G. Matten, M. Ouisse, and E. Rivet, Broadband Nonreciprocal Acoustic Propagation Using Programmable Boundary Conditions: From Analytical Modeling to Experimental Implementation, Phys. Rev. Appl. 12, 054033 (2019).

[19] C. Yangyang, X. Li, G. Hu, M. Haberman, and G. Huang, An active mechanical Willis meta-layer with asymmetric polarizabilities, Nature Comm. 11 (2020).

[20] N. Geib, A. Sasmal, Z. Wang, Y. Zhai, B.-I. Popa, and K. Grosh, Tunable nonlocal purely active nonreciprocal acoustic media, Phys. Rev. B 103, 165427 (2021).

[21] H. Lissek, R. Boulandet, and R. Fleury, Electroacoustic absorbers: Bridging the gap between shunt loudspeakers and active sound absorption, J. Acoust. Soc. Am. 129, 2968 (2011).

[22] R. Fleury, D. Sounas, and A. Alù, An invisible acoustic sensor based on parity-time symmetry, Nature comm. 6, 5905 (2015).

[23] T. T. Koutserimpas, E. Rivet, H. Lissek, and R. Fleury, Active Acoustic Resonators with Reconfigurable Resonance Frequency, Absorption, and Bandwidth, Phys. Rev. Appl. 12, 054064 (2019).

[24] E. Rivet, A. Brandstötter, K. Makris, H. Lissek, S. Rotter, and R. Fleury, Constant-pressure sound waves in non-Hermitian disordered media, Nature Phys. 14 (2018).

[25] A. Pierce, Acoustics: An Introduction to Its Physical Principles and Applications (Acoustical Society of America, 1989).

[26] G. Lemarquand and B. Merit, Magnetic structure for an ironless electrodynamic loudspeaker motor, motors, and loudspeakers, US Patent 8,055,010 B2 (2011).

[27] J. d'Appolito, Testing Loudspeakers (Audio Amateur Inc, Peterborough, 2018).

[28] M. Munjal and A. Doige, Theory of a two source-location method for direct experimental evaluation of the fourpole parameters of an aeroacoustic element, J. Sound Vib. 141, 323 (1990).

[29] H. Boden and M. Abom, Influence of errors on the twomicrophone method for measuring acoustic properties in ducts, J. Acoust. Soc. Am. 79, 541 (1986).

[30] A. Malvino and D. Bates, Electronic Principles (Macgraw-Hill, 2015). 